\title{
One-loop electroweak radiative corrections to polarized Bhabha scattering
}

\author{
D. Bardin, ${ }^{*}$ Ya. Dydyshka, L. Kalinovskaya, L. Rumyantsev, ${ }^{\dagger}$ and R. Sadykov \\ Dzhelepov Laboratory of Nuclear Problems, JINR, Dubna 141980, Russia \\ A. Arbuzov ${ }^{\ddagger}$ and S. Bondarenko ${ }^{\S}$ \\ Bogoliubov Laboratory of Theoretical Physics, JINR, Dubna 141980, Russia
}

(Received 17 March 2018; published 2 July 2018)

\begin{abstract}
Theoretical predictions for Bhabha scattering observables are presented including complete one-loop electroweak radiative corrections. Longitudinal polarization of the initial beams is taken into account. Numerical results for the asymmetry $A_{L R}$ and the relative correction $\delta$ are given for the set of future $e^{+} e^{-}$ collider energies $E_{\mathrm{cm}}=250,500,1000 \mathrm{GeV}$ with various polarization degrees.
\end{abstract}

DOI: 10.1103/PhysRevD.98.013001

\section{INTRODUCTION}

The complete one-loop electroweak (EW) corrections to unpolarized Bhabha scattering [1] have been thoroughly studied for many years by the authors of [2] and later the authors of [3-12]. The Bhabha cross section with the oneloop QED contribution including transverse and longitudinal polarizations of the incoming beams is presented in $[13,14]$. Many Monte Carlo event generators for Bhabha scattering were created; see, e.g., [15] and references therein. In our review [16] we have presented the SANC modules for the one-loop electroweak radiative corrections (RC) for Bhabha scattering: the helicity amplitudes (HA) and form factors (FF).

As compared with hadronic collisions being studied at the LHC, $e^{+} e^{-}$interaction processes have a clean initial state and a much lower multiplicity, and therefore provide a higher measurement precision in most cases. The substantially higher energy range of the future colliders also demands reestimation of various effects from both experimental and theoretical sides. Precise measurements with polarized beams at the future $e^{+} e^{-}$colliders ILC [17] and CLIC [18] definitely require modern advanced theoretical support [19-22]. In particular, physical programs of the future $e^{+} e^{-}$linear colliders [23-25] always demonstrated a great interest in the effects related to the beam polarization.

\footnotetext{
*Deceased.

${ }^{\dagger}$ Also at Institute of Physics, Southern Federal University, Rostov-on-Don 344090, Russia.

Also at Dubna University, Dubna 141980, Russia.

§bondarenko@jinr.ru

Published by the American Physical Society under the terms of the Creative Commons Attribution 4.0 International license. Further distribution of this work must maintain attribution to the author(s) and the published article's title, journal citation, and DOI. Funded by SCOAP ${ }^{3}$.
}

In this article we present the complete one-loop calculation of the EW radiative corrections to Bhabha scattering with polarized beams. Numerical estimates are shown for the correction to the differential distribution in the cosine of the electron scattering angle. The relevant contributions to the cross section are calculated analytically and then evaluated numerically.

In order to verify our results, we performed several tuned comparisons with the results of the alternative systems where available. The sum of virtual and soft photon bremsstrahlung contributions in the unpolarized case are compared with the AITALC-1.4 code [12]. The polarized Born and hard photon bremsstrahlung contributions are compared with the corresponding values obtained with the help of the WHIZARD code $[20,26,27]$. The unpolarized hard photon contribution is compared analytically with the result of the CALCHEP code [28].

This paper is organized as follows. Section II, the main section of this paper, is fully devoted to the cross section calculation technique at the one-loop level. Expressions for covariant and helicity amplitudes are presented. The approach for treatment of polarization effects is discussed. Section III contains numerical results for the asymmetry $A_{L R}$ and for the relative correction $\delta$ to the differential cross section. Comparisons with the results of other codes are also presented. Finally, in Sec. IV we conclude with a discussion of the obtained results.

\section{DIFFERENTIAL CROSS SECTION}

Let us consider the scattering of longitudinally polarized $e^{+}$and $e^{-}$beams with the four momenta $p_{1}$ and $p_{2}$ for the incoming particles and $p_{3}$ and $p_{4}$ for the outgoing ones:

$$
e^{+}\left(p_{1}\right)+e^{-}\left(p_{2}\right) \rightarrow e^{-}\left(p_{3}\right)+e^{+}\left(p_{4}\right) .
$$



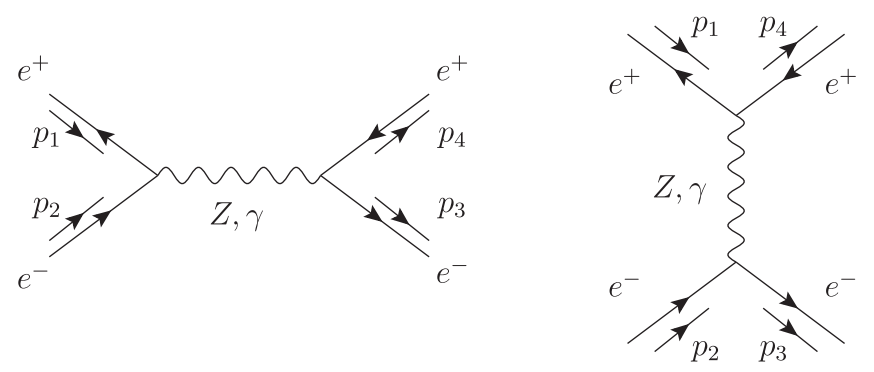

FIG. 1. The $s$ (left) and $t$ (right) channels of the Bhabha process at the lowest order.

Where it is possible, we work in the massless limit and neglect the effects suppressed by the ratio of the electron mass to the beam energy. The Feynman diagrams for the Born level are shown in Fig. 1.

For the differential cross section $e^{+} e^{-} \rightarrow e^{-} e^{+}$one gets

$$
d \sigma=\frac{1}{32 \pi s} \overline{|\mathcal{A}|^{2}} d \cos \vartheta
$$

where $\mathcal{A}$ is the covariant amplitude (CA) of the process, $\sqrt{s} / 2$ is the electron energy, and $\vartheta$ is the scattering angle in the center-of-mass system (CMS).

\section{A. Covariant one-loop amplitude}

The one-loop covariant amplitude comes from the straightforward standard calculation by means of SANC programs and procedures of all diagrams contributing to a given process at the tree (Born) and one-loop levels. The amplitude contains kinematic factors and coupling constants. It is parametrized by a certain number of FFs, which are denoted by $\mathcal{F}$ in general with an index labeling the corresponding Lorentz tensor structure. The number of FFs is equal to the number of the relevant structures. For the processes with nonzero tree-level amplitudes the FFs have the form

$$
\mathcal{F}=1+k \tilde{\mathcal{F}}
$$

where " 1 " stands for the Born level and the term $\tilde{\mathcal{F}}$ with the factor

$$
k=\frac{g^{2}}{16 \pi^{2}}
$$

is due to one-loop corrections. After squaring the amplitude we neglect terms proportional to $k^{2}$ in order to get the pure one-loop approximation without any admixture of higherorder terms that can be added later.

The CA for Bhabha scattering can be written (if the electron mass is neglected and because of the symmetry of the process: $\mathcal{F}_{L Q}=\mathcal{F}_{Q L}$ ) by the electromagnetic running coupling constant and four FFs with permuted arguments $s$ and $t$ as

$$
\begin{aligned}
\mathcal{A}= & \mathcal{A}_{\gamma}(s)+\mathcal{A}_{Z}(s)-\left[\mathcal{A}_{\gamma}(t)+\mathcal{A}_{Z}(t)\right] \\
= & i e^{2}\left\{\left[\gamma_{\mu} \otimes \gamma_{\mu} \frac{\mathcal{F}_{\gamma}(s)}{s}-\gamma_{\mu} \otimes \gamma_{\mu} \frac{\mathcal{F}_{\gamma}(t)}{t}\right]\right. \\
& +\frac{\chi_{Z}(s)}{s}\left\{\left(I_{e}^{(3)}\right)^{2} \gamma_{\mu} \gamma_{6} \otimes \gamma_{\mu} \gamma_{6} \mathcal{F}_{L L}(s, t, u)\right. \\
& \left.+2 \delta_{e} I_{e}^{(3)} \gamma_{\mu} \otimes \gamma_{\mu} \gamma_{6} \mathcal{F}_{Q L}(s, t, u)+\delta_{e}^{2} \gamma_{\mu} \otimes \gamma_{\mu} \mathcal{F}_{Q Q}(s, t, u)\right\} \\
& -\frac{\chi_{Z}(t)}{t}\{s \leftrightarrow t\},
\end{aligned}
$$

where $\gamma_{6}=\left(1+\gamma_{5}\right)$, the electron charge is $Q_{e}$, and couplings $I_{e}^{(3)}, \delta_{e}=v_{e}-a_{e}$. The symbol $\otimes$ is used in the following shorthand notation:

$$
J_{\mu}^{i} \otimes J_{\mu}^{j}=\bar{v}\left(i, p_{1}\right) J_{\mu}^{i} u\left(i, p_{2}\right) \bar{u}\left(j, p_{3}\right) J_{\mu}^{j} v\left(j, p_{4}\right)
$$

for the $s$ channel and

$$
J_{\mu}^{i} \otimes J_{\mu}^{j}=\bar{u}\left(i, p_{3}\right) J_{\mu}^{i} u\left(i, p_{2}\right) \bar{v}\left(j, p_{1}\right) J_{\mu}^{j} v\left(j, p_{4}\right)
$$

for the $t$ channel. The function $\chi_{Z}$ is

$$
\chi_{Z}(I)=\frac{1}{4 s_{W}^{2} c_{W}^{2}} \frac{I}{I-M_{Z}^{2}+i M_{Z} \Gamma_{Z}},
$$

with $I=s$ or $t$. In the $t$ channel $\Gamma_{Z}$ is equal to zero.

\section{B. Virtual, soft, and hard contributions}

The complete result for $\mathcal{O}(\alpha)$ corrections can be separated into the virtual (loop) contribution, the part due to the soft photon emission, and the last one due to the real hard photon Bremsstrahlung.

\section{Born and virtual modules}

Our main approach is to calculate a cross section by squaring noninterfering HAs. In the CA approach we derive tensor structures and FFs. The next step is the projection to the helicity basis. As the result one gets a set of noninterfering amplitudes, since all of them are characterized by different sets of helicity quantum numbers.

In this subsection we collect the analytic expressions for the HAs. There are six nonzero HAs, however, since for Bhabha scattering $\mathcal{F}_{L Q}^{Z}=\mathcal{F}_{Q L}^{Z}$, the number of independent HAs is actually reduced to four as expected.

We obtain the compact expression for the Born $\left(\mathcal{F}_{Q L, L L, Q Q}=1\right)$ and the virtual part by HA approach: 


$$
\begin{aligned}
\mathcal{H}_{++++}= & \mathcal{H}_{----} \\
= & -2 e^{2} \frac{s}{t}\left[\mathcal{F}_{Q Q}^{(\gamma, Z)}(t, s, u)-\chi_{Z}(t) \delta_{e} \mathcal{F}_{Q L}^{Z}(t, s, u)\right], \\
\mathcal{H}_{+-+-}= & \mathcal{H}_{-+-+} \\
= & e^{2} c_{-}\left[\mathcal{F}_{Q Q}^{(\gamma, Z)}(s, t, u)-\chi_{Z}(s) \delta_{e} \mathcal{F}_{L Q}^{Z}(s, t, u)\right], \\
\mathcal{H}_{+--+}= & -e^{2} c_{+}\left(\left[\mathcal{F}_{Q Q}^{(\gamma, Z)}(s, t, u)+\chi_{Z}(s)\left(\mathcal{F}_{L L}^{Z}(s, t, u)\right.\right.\right. \\
& \left.\left.\left.-2 \delta_{e} \mathcal{F}_{L Q}^{Z}(s, t, u)\right)\right]+\frac{s}{t}[s \leftrightarrow t]\right), \\
\mathcal{H}_{-++-}= & -e^{2} c_{+}\left(\left[\mathcal{F}_{Q Q}^{(\gamma, Z)}(s, t, u)\right]+\frac{s}{t}[s \leftrightarrow t]\right),
\end{aligned}
$$

where $c_{ \pm}=\cos \vartheta \pm 1$ and $\mathcal{F}_{Q Q}^{(\gamma, Z)}(a, b, c)=\mathcal{F}_{Q Q}^{(\gamma)}(a, b, c)+$ $\chi_{Z}(a) \delta_{e}^{2} \mathcal{F}_{Q Q}^{(Z)}(a, b, c)$.

\section{Bremsstrahlung module}

The bremsstrahlung module of the SANC system computes the contributions due to the soft and inclusive hard real photon emission. The soft photon contribution contains infrared divergences and has to compensate the corresponding divergences of the one-loop virtual QED corrections.

The soft photon Bremsstrahlung correction can be calculated analytically. It is factorized in front of the Born cross section. It depends on the auxiliary parameter which separates kinematical domains of the soft and hard photon emission in a given reference frame. The polarization dependence is contained in $\sigma^{\text {Born }}$. The explicit form for the soft photon contribution is

$$
\begin{aligned}
\sigma^{\text {soft }}= & -\sigma^{\text {Born }} \frac{\alpha}{\pi} Q_{e}^{2}\left\{\left(1+\ln \left(\frac{m_{e}^{2}}{s}\right)\right)^{2}\right. \\
& +\ln \left(-\frac{u}{s}\right)^{2}-\ln \left(-\frac{t}{s}\right)^{2}-2 \operatorname{Li}_{2}\left(-\frac{u}{s}\right) \\
& +2 \operatorname{Li}_{2}\left(-\frac{t}{s}\right)+4 \operatorname{Li}_{2}(1)-1 \\
& \left.+2 \ln \left(\frac{4 \omega^{2}}{\lambda}\right)\left[1+\ln \left(\frac{m_{e}^{2}}{s}\right)-\ln \left(\frac{t}{u}\right)\right]\right\} .
\end{aligned}
$$

Here $\omega$ is the soft-hard separator, $\lambda$ is an auxiliary infinitesimal photon mass, and the Spence function $\mathrm{Li}_{2}(z)=-\int_{0}^{z} \frac{\ln (1-x)}{x} d x$.

The contribution of the hard real photon emission is obtained by direct squaring of the matrix element. Explicit formulas for the differential distribution of the Bhabha process with hard photon emission are too long to be listed here.

\section{Longitudinal polarization}

To study the case of the longitudinal polarization, we produce the helicity amplitudes and make a formal application of Eq. (1.15) from [29]. In our notation the corresponding cross section of Bhabha scattering with the longitudinal polarization can be expressed as

$$
\begin{aligned}
\frac{d \sigma\left(P_{e^{-}}, P_{e^{+}}\right)}{d \cos \vartheta}= & \frac{1}{128 \pi s}\left[\left(1+P_{e^{-}}\right)\left(1+P_{e^{+}}\right) \sum_{i j}\left|\mathcal{H}_{++i j}\right|^{2}\right. \\
& +\left(1-P_{e^{-}}\right)\left(1+P_{e^{+}}\right) \sum_{i j}\left|\mathcal{H}_{+-i j}\right|^{2} \\
& +\left(1+P_{e^{-}}\right)\left(1-P_{e^{+}}\right) \sum_{i j}\left|\mathcal{H}_{-+i j}\right|^{2} \\
& \left.+\left(1-P_{e^{-}}\right)\left(1-P_{e^{+}}\right) \sum_{i j}\left|\mathcal{H}_{--i j}\right|^{2}\right] .
\end{aligned}
$$

For the cross-check we got analytical zero for the difference between the square of the covariant amplitude (we introduced the spin density matrix into our procedures) and Eq. (11).

The asymmetry $A_{L R}$ and the relative correction $\delta$ are defined as

$$
\begin{aligned}
A_{L R} & =\frac{d \sigma(-1,1)-d \sigma(1,-1)}{d \sigma(-1,1)+d \sigma(1,-1)}, \\
\delta & =\frac{d \sigma^{1-\operatorname{loop}}\left(P_{e^{-}}, P_{e^{+}}\right)}{d \sigma^{\text {Born }}\left(P_{e^{-}}, P_{e^{+}}\right)}-1,
\end{aligned}
$$

where we omitted $d \cos \vartheta$ for shortness.

\section{NUMERICAL RESULTS AND COMPARISONS}

In this section, we present numerical results for $\mathrm{EW} \mathrm{RC}$ to Bhabha scattering obtained by means of the SANC Monte Carlo event generator. Comparisons of our results for specific contributions with the ones existing in the literature are also given.

There are many studies devoted to the Bhabha process; see, e.g., [12] and references therein. It is highly nontrivial to realize a tuned comparison of the numerical results, since authors usually do not present the complete list of the input parameters and do not specify the calculation scheme. Eventually, we compare with the modern packages AITALC and WHIZARD that allow one to choose the following common set of the input parameters: 
TABLE I. Tuned comparison of the SANC and WHIZARD results for the Born and hard bremsstrahlung contributions to polarized Bhabha scattering for $\sqrt{s}=250,500$, and $1000 \mathrm{GeV}$.

\begin{tabular}{lllll}
\hline \hline$P_{e^{-}}, P_{e^{+}}$ & \multicolumn{5}{c}{0,0} & \multicolumn{3}{c}{$-0.8,0$} & $-0.8,-0.6$ & $-0.8,0.6$ \\
\hline \multicolumn{5}{c}{$\sqrt{s}=250 \mathrm{GeV}$} \\
$\sigma^{\text {Born }}, \mathrm{pb}$ & $56.677(1)$ & $57.774(1)$ & $56.272(1)$ & $59.276(1)$ \\
$\sigma^{\text {Born }}, \mathrm{pb}$ & $56.677(1)$ & $57.775(1)$ & $56.272(1)$ & $59.275(1)$ \\
$\sigma^{\text {hard }}, \mathrm{pb}$ & $48.62(1)$ & $49.58(1)$ & $48.74(1)$ & $50.40(1)$ \\
$\sigma^{\text {hard }}, \mathrm{pb}$ & $48.65(1)$ & $49.56(1)$ & $48.78(1)$ & $50.44(1)$ \\
& \multicolumn{5}{c}{$\sqrt{s}=500 \mathrm{GeV}$} \\
$\sigma^{\text {Born }}, \mathrm{pb}$ & $14.379(1)$ & $15.030(1)$ & $12.706(1)$ & $17.355(1)$ \\
$\sigma^{\text {Born }}, \mathrm{pb}$ & $14.379(1)$ & $15.030(1)$ & $12.706(1)$ & $17.354(1)$ \\
$\sigma^{\text {hard }}, \mathrm{pb}$ & $15.14(1)$ & $15.81(1)$ & $13.54(1)$ & $18.07(1)$ \\
$\sigma^{\text {hard }}, \mathrm{pb}$ & $15.12(1)$ & $15.79(1)$ & $13.55(1)$ & $18.11(2)$ \\
& \multicolumn{5}{c}{$\sqrt{s}=1000 \mathrm{GeV}$} \\
$\sigma^{\text {Born }}, \mathrm{pb}$ & $3.6792(1)$ & $3.9057(1)$ & $3.0358(1)$ & $4.7756(1)$ \\
$\sigma^{\text {Born }}, \mathrm{pb}$ & $3.6792(1)$ & $3.9057(1)$ & $3.0358(1)$ & $4.7755(1)$ \\
$\sigma^{\text {hard }}, \mathrm{pb}$ & $4.693(1)$ & $4.976(1)$ & $3.912(1)$ & $6.041(1)$ \\
$\sigma^{\text {hard }}, \mathrm{pb}$ & $4.694(1)$ & $4.975(1)$ & $3.913(1)$ & $6.043(2)$ \\
\hline \hline
\end{tabular}

$$
\begin{aligned}
\alpha^{-1}(0) & =137.03599976, & & \\
M_{W} & =80.4514958 \mathrm{GeV}, & M_{Z} & =91.1876 \mathrm{GeV}, \\
\Gamma_{Z} & =2.49977 \mathrm{GeV}, & m_{e} & =0.51099907 \mathrm{MeV}, \\
m_{\mu} & =0.105658389 \mathrm{GeV}, & m_{\tau} & =1.77705 \mathrm{GeV}, \\
m_{d} & =0.083 \mathrm{GeV}, & m_{s} & =0.215 \mathrm{GeV}, \\
m_{b} & =4.7 \mathrm{GeV}, & m_{u} & =0.062 \mathrm{GeV}, \\
m_{c} & =1.5 \mathrm{GeV}, & m_{t} & =173.8 \mathrm{GeV} . \quad(13)
\end{aligned}
$$

The $\alpha(0)$ EW scheme is used in all calculations.

All results are obtained for the set of the energy $E_{\mathrm{cm}}=250,500$, and $1000 \mathrm{GeV}$ for the following magnitudes of the electron $\left(P_{e^{-}}\right)$and the positron $\left(P_{e^{+}}\right)$beam polarizations: $(0,0),(-0.8,0),(-0.8,-0.6)$, $(-0.8,0.6)$.

\section{A. Comparison for Born and hard photon contributions}

First of all, we verified an agreement between our analytic result for the unpolarized hard photon bremsstrahlung process cross section with the one obtained with the help of the CALCHEP system [28].

The numerical comparison for the hard photon bremsstrahlung cross section with polarized initial particles is performed using the WHIZARD system. Table I shows the good agreement between the SANC results (the second row) for the Born and hard photon Bremsstrahlung contributions with the ones obtained with the help of the WHIZARD (the first row) program [30]. The range of scattering angles for the final electrons and positrons in this comparison was limited by the condition $|\cos \theta|<0.9$ with the additional condition $E_{\gamma} \geq 1 \mathrm{GeV}$ for the emitted photon energy.

\section{B. Comparison of virtual and soft photon bremsstrahlung contributions}

We obtained a very good agreement (six significant digits) in the comparison of the SANC and AITALC-1.4 [12] results for the unpolarized differential Born cross section and for the sum of the virtual and the soft photon bremsstrahlung contributions. The comparison was done for the different values of the scattering angles $(\cos \vartheta$ : from -0.9 up to +0.9999 ).

\section{Results for Born and one-loop cross section}

The unpolarized differential cross section of Bhabha scattering and the relative $\mathcal{O}(\alpha)$ correction $\delta$ (in percent) as a function of the electron scattering angle are shown in Figs. 2-4 for $|\cos \theta|<0.9$ and different CMS energies.

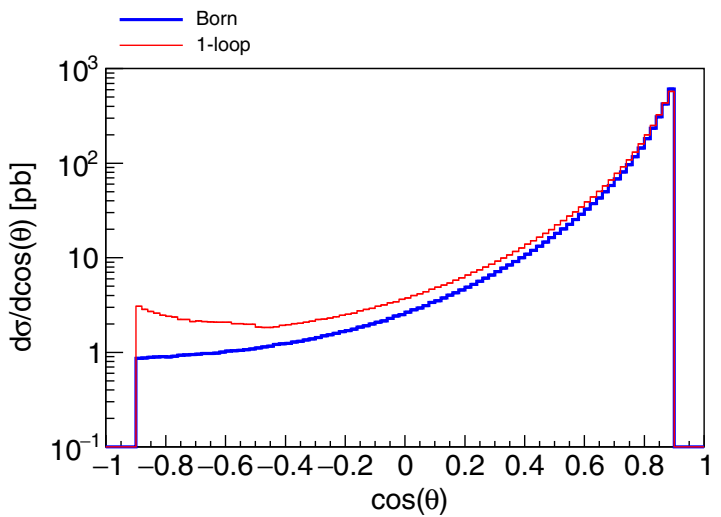

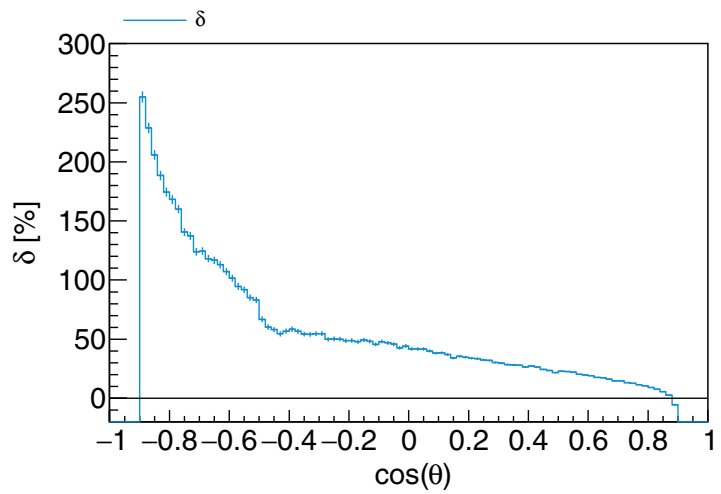

FIG. 2. The differential cross section (left) [in pb] and the relative correction $\delta$ (right) [in \%] vs the cosine of the electron scattering angle for $\sqrt{s}=250 \mathrm{GeV}$. 

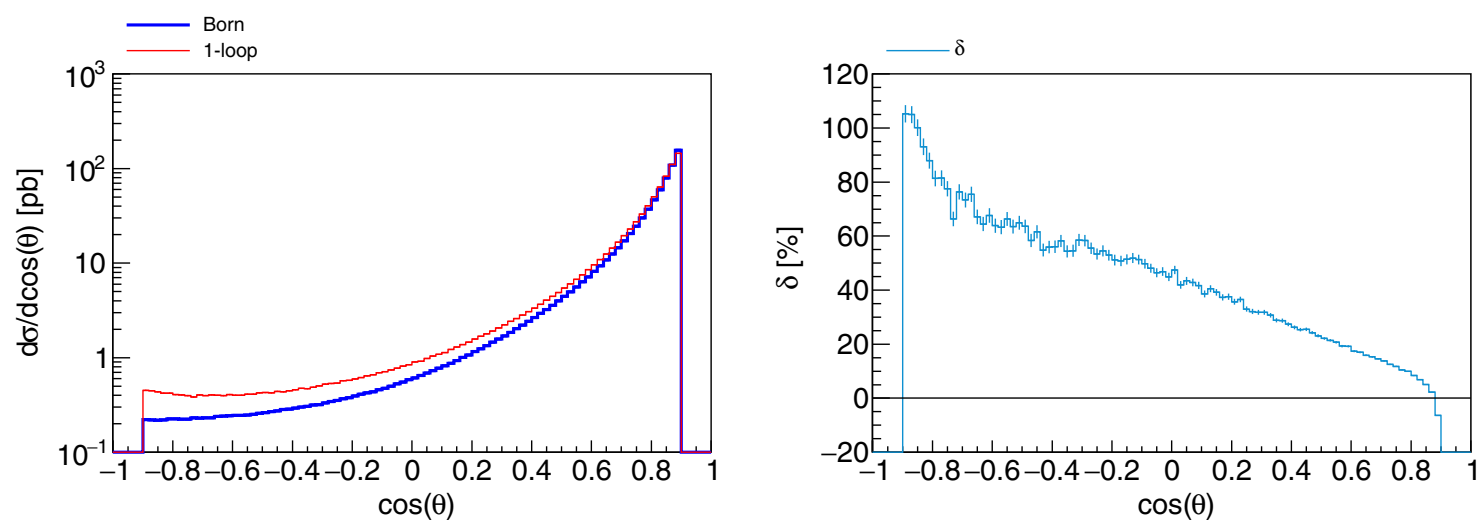

FIG. 3. The differential cross section (left) [in pb] and the relative correction $\delta$ (right) [in \%] vs the cosine of the electron scattering angle for $\sqrt{s}=500 \mathrm{GeV}$.
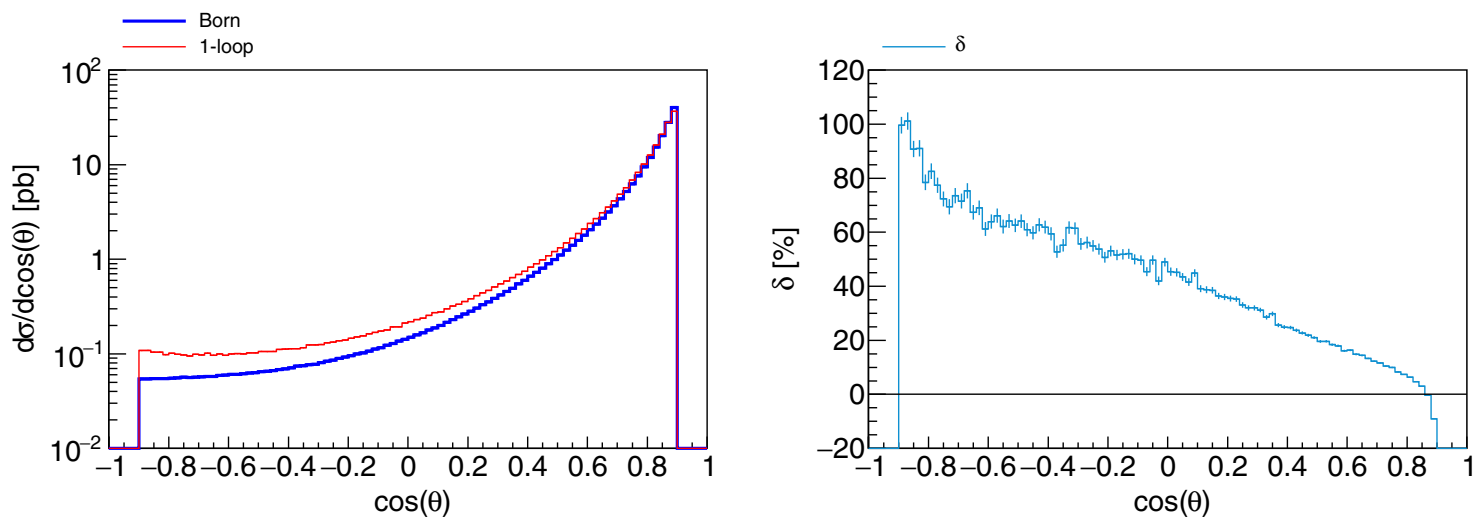

FIG. 4. The differential cross section (left) [in pb] and the relative correction $\delta$ (right) [in \%] vs the cosine of the electron scattering angle for $\sqrt{s}=1000 \mathrm{GeV}$.

TABLE II. Born and one-loop cross sections of Bhabha scattering and the corresponding relative corrections $\delta$ for $\sqrt{s}=250,500$, and $1000 \mathrm{GeV}$.

\begin{tabular}{lcccc}
\hline \hline$P_{e^{-}}, P_{e^{+}}$ & 0,0 & $-0.8,0$ & $-0.8,-0.6$ & $-0.8,0.6$ \\
\hline \multicolumn{5}{c}{$\sqrt{s}=250 \mathrm{GeV}$} \\
$\sigma_{e^{+} e^{-}}^{\text {Born }}, \mathrm{pb}$ & $56.6763(1)$ & $57.7738(1)$ & $56.2725(4)$ & $59.2753(5)$ \\
$\sigma_{e^{+} e^{-}}, \mathrm{pb}$ & $61.731(6)$ & $62.587(6)$ & $61.878(6)$ & $63.287(7)$ \\
$\delta, \%$ & $8.92(1)$ & $8.33(1)$ & $9.96(1)$ & $6.77(1)$ \\
\multicolumn{5}{c}{$\sqrt{s}=500 \mathrm{GeV}$} \\
$\sigma_{e^{+} e^{-}}^{\text {Born }}, \mathrm{pb}$ & $14.3789(1)$ & $15.0305(1)$ & $12.7061(1)$ & $17.3550(2)$ \\
$\sigma_{e^{+} e^{-}}^{1-\text { loop }}, \mathrm{pb}$ & $15.465(2)$ & $15.870(2)$ & $13.861(1)$ & $17.884(2)$ \\
$\delta, \%$ & $7.56(1)$ & $5.59(1)$ & $9.09(1)$ & $3.05(1)$ \\
\multicolumn{5}{c}{$\sqrt{s}=1000 \mathrm{GeV}$} \\
$\sigma_{e^{+} e^{-}}^{\text {Born }}, \mathrm{pb}$ & $3.67921(1)$ & $3.90568(1)$ & $3.03577(3)$ & $4.77562(5)$ \\
$\sigma_{e^{+} e^{-}}^{1-\text { loop }}, \mathrm{pb}$ & $3.8637(4)$ & $3.9445(4)$ & $3.2332(3)$ & $4.6542(7)$ \\
$\delta, \%$ & $5.02(1)$ & $0.99(1)$ & $6.50(1)$ & $-2.54(1)$ \\
\hline \hline
\end{tabular}

The huge relative radiative corrections for the backward scattering angles are due to the smallness of the Born cross section in this domain, which does not mean any problem with the perturbation theory.

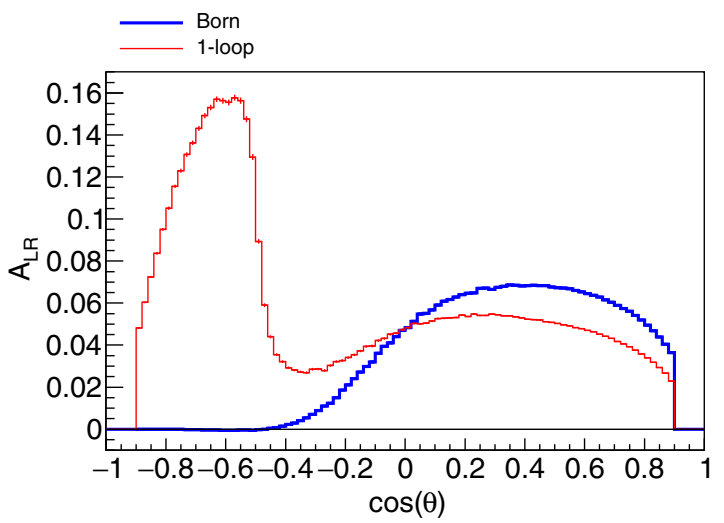

FIG. 5. The left-right asymmetry $A_{L R}$ as a function of the cosine of the electron scattering angle at $\sqrt{s}=250 \mathrm{GeV}$. 


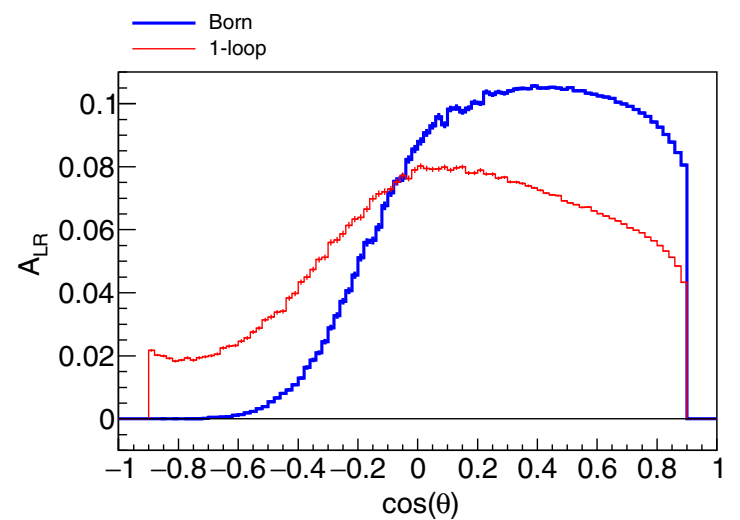

FIG. 6. The left-right asymmetry $A_{L R}$ as a function of the cosine of the electron scattering angle at $\sqrt{s}=500 \mathrm{GeV}$.

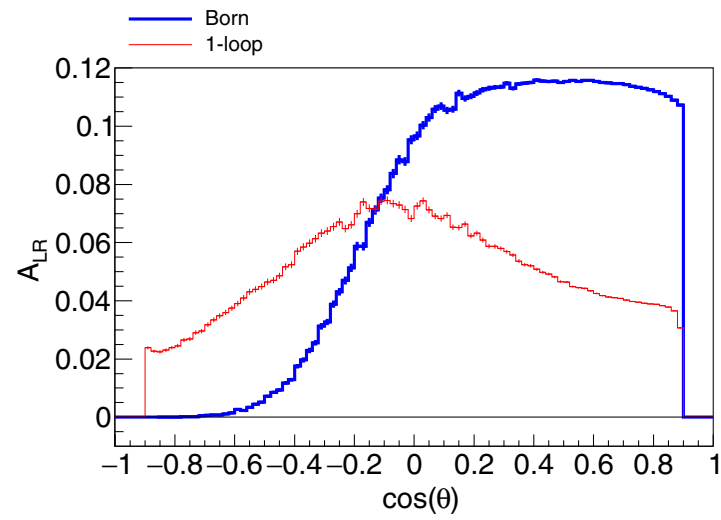

FIG. 7. The left-right asymmetry $A_{L R}$ as a function of the cosine of the electron scattering angle at $\sqrt{s}=1000 \mathrm{GeV}$.

The integrated cross section of the Bhabha scattering and the relative correction $\delta$ are given in Table II for various energies and beam polarization degrees.

The $A_{L R}$ asymmetry at $\sqrt{s}=250,500$, and $1000 \mathrm{GeV}$ is shown in Figs. 5-7. One can see that the EW radiative corrections affect the asymmetry very strongly.

\section{CONCLUSIONS}

The theoretical description of Bhabha scattering with taking into account the radiative corrections is crucial for the high-precision measurement of this process and thus for luminosity monitoring at the modern and future $e^{+} e^{-}$ colliders. Consideration of the beam polarization is a novel requirement for the theoretical predictions for the $e^{+} e^{-}$collisions at the energies of CLIC and ILC. Moreover, our results can be relevant for the physical program of the Super $c-\tau$ factory [31] planned to be built in Novosibirsk, where polarization of the electron beam is also foreseen.

We show that the complete $\mathcal{O}(\alpha)$ electroweak radiative corrections provide a considerable impact on the differential cross section and the left-right asymmetry. Moreover, the corrections themselves are rather sensitive to polarization degrees of the initial beams.

The observed magnitude of the first order corrections certainly provokes the question about the size of the higherorder corrections to this process. Some of those corrections are known in the literature (but mainly the pure QED ones for the unpolarized case). To estimate the theoretical uncertainty we plan to implement the known higher-order corrections to Bhabha scattering into our Monte Carlo event generator.

\section{ACKNOWLEDGMENTS}

We express our sincere gratitude to Sabine Riemann [32] for fruitful stimulating discussions. We thank Gizo Nanava for collaboration on the SANC system. Results were obtained within the framework of State Program No. 3.9696.2017/8.9 from the Ministry of Education and Science of Russia. We are grateful to Dr. A. Gladyshev for the help in the preparation of the manuscript.
[1] H. J. Bhabha, The scattering of positrons by electrons with exchange on Dirac's theory of the positron, Proc. R. Soc. A 154, 195 (1936).

[2] M. Consoli, One-loop corrections to $e^{+} e^{-} \rightarrow e^{+} e^{-}$in the Weinberg model, Nucl. Phys. B160, 208 (1979).

[3] M. Bohm, A. Denner, W. Hollik, and R. Sommer, Radiative Corrections to Bhabha Scattering in SU(2) X U(1), Phys. Lett. 144B, 414 (1984).

[4] K. Tobimatsu and Y. Shimizu, Radiative Correction to $e^{+} e^{-} \rightarrow e^{+} e^{-}$in the Electroweak Theory. II: Corrected
Elastic Cross Section and Positron Energy Spectrum, Prog. Theor. Phys. 75, 905 (1986).

[5] M. Bohm, A. Denner, and W. Hollik, Radiative corrections to Bhabha scattering at high energies (I): Virtual and soft photon corrections, Nucl. Phys. B304, 687 (1988).

[6] F. A. Berends, R. Kleiss, and W. Hollik, Radiative corrections to Bhabha scattering at high energies: (II). Hard photon corrections and Monte Carlo treatment, Nucl. Phys. B304, 712 (1988). 
[7] S. Kuroda, T. Kamitani, K. Tobimatsu, S. Kawabata, and Y. Shimizu, Bhabha scattering at high energy, Comput. Phys. Commun. 48, 335 (1988).

[8] D. Yu. Bardin, W. Hollik, and T. Riemann, Bhabha scattering at high energy, Z. Phys. C 49, 485 (1991).

[9] W. Beenakker, F. A. Berends, and S. C. van der Marck, Large-angle Bhabha scattering, Nucl. Phys. B349, 323 (1991).

[10] W. Beenakker, F. A. Berends, and S. C. van der Marck, Small-angle Bhabha scattering, Nucl. Phys. B355, 281 (1991).

[11] G. Montagna, F. Piccinini, O. Nicrosini, G. Passarino, and R. Pittau, On a semi-analytical and realistic approach to $e^{+} e^{-}$annihilation into fermion pairs and to Bhabha scattering within the minimal standard model at LEP energies, Nucl. Phys. B401, 3 (1993).

[12] J. Fleischer, J. Gluza, A. Lorca, and T. Riemann, One-loop photonic corrections to Bhabha scattering in $d=4-2 \varepsilon$ dimensions, Eur. J. Phys. C 48, 35 (2006).

[13] W. Hollik and A. Zepeda, Polarized Bhabha scattering in multiboson electroweak gauge models, Z. Phys. C 12, 67 (1982).

[14] W. Hollik, Higher order QED contributions to polarized Bhabha scattering, Phys. Lett. 123B, 259 (1983).

[15] S. Jadach et al., Event generators for Bhabha scattering, in CERN Workshop on LEP2 Physics (followed by 2 nd meeting, 15-16 June 1995 and 3rd meeting 2-3 November 1995) Geneva, Switzerland, 1995 (CERN, Geneva, 1996), pp. 229-298.

[16] A. Andonov, A. Arbuzov, D. Bardin, S. Bondarenko, P. Christova, L. Kalinovskaya, G. Nanava, and W. von Schlippe, SANCscope-v.1.00, Comput. Phys. Commun. 174, 481 (2006); Erratum 177, 623 (2007).

[17] ILC, https://www.linearcollider.org/ILC.

[18] CLIC, http://clic-study.web.cern.ch.

[19] P. H. Khiem et al., Full $\mathcal{O}(\alpha)$ electroweak radiative corrections to $t \bar{t} \gamma$ and $e^{-} e^{+} \gamma$ productions at ILC with GRACELoop, in Proceedings, 9th Rencontres du Vietnam: Windows on the Universe: Quy Nhon, Vietnam, 2013 (Thé Gioi, 2013), pp. 261-264.
[20] T. Ohl, WHiZard and O'Mega, in Proceedings, LoopFest V: Radiative Corrections for the International Linear Collider: Multi-loops and Multi-legs: SLAC, Menlo Park, California, 2006, http://www-public.slac.stanford.edu/sciDoc/docMeta .aspx?slacPubNumber=slac-wp-071.

[21] C. M. Carloni Calame, G. Montagna, O. Nicrosini, and F. Piccinini, High-precision Luminosity at $e^{+} e^{-}$Colliders: Theory Status and Challenges, Acta Phys. Pol. B 46, 2227 (2015).

[22] S. Riemann, Status and Prospects of $e^{+} e^{-}$Linear Collider Projects, Acta Phys. Pol. B 46, 2213 (2015).

[23] E. Accomando et al. (ECFA/DESY LC Physics Working Group), Physics with $e^{+} e^{-}$linear colliders, Phys. Rep. 299, 1 (1998).

[24] E. Accomando et al. (CLIC Physics Working Group), Physics at the CLIC multi-TeV linear collider, in Proceedings, 11th International Conference on Hadron spectroscopy (Hadron 2005): Rio de Janeiro, Brazil, 2005 [AIP Conf. Proc. 814, 1].

[25] A. Arbey et al., Physics at the $e^{+} e^{-}$linear collider, Eur. Phys. J. C 75, 371 (2015).

[26] W. Kilian, T. Ohl, and J. Reuter, WHIZARD—simulating multi-particle processes at LHC and ILC, Eur. Phys. J. C 71, 1742 (2011).

[27] W. Kilian, F. Bach, T. Ohl, and J. Reuter, WHIZARD 2.2 for Linear Colliders, in International Workshop on Future Linear Colliders (LCWS13) Tokyo, Japan, 2013 (The University of Tokyo, Tokyo, 2014).

[28] A. Belyaev, N. D. Christensen, and A. Pukhov, CalcHEP 3.4 for collider physics within and beyond the Standard Model, Comput. Phys. Commun. 184, 1729 (2013).

[29] G. Moortgat-Pick et al., Polarized positrons and electrons at the linear collider, Phys. Rep. 460, 131 (2008).

[30] T. Ohl, O’Mega \& WHIZARD: Monte Carlo event generator generation for future colliders, AIP Conf. Proc. 578, 638 (2001).

[31] S. Eidelman, Project of the Super-tau-charm Factory in Novosibirsk, Nucl. Part. Phys. Proc. 260, 238 (2015).

[32] S. Riemann, Precision measurements with polarized beams, Calc-2015 report, https://goo.gl/a8mtqh. 